\title{
Globale Ökonomie jenseits dünner Beschreibungen
}

\author{
Erste Überlegungen zu Vertrauen im neuen Markt für \\ Mikrofinanzen
}

Barbara Grimpe

\section{Problemstellung}

In vielen sogenannten «Entwicklungsländern» rund um die Welt gibt es Mikrofinanzprojekte: Kleinunternehmer und -unternehmerinnen erhalten von örtlichen Mikrofinanzinstitutionen Mikrokredite, die ihrerseits oftmals aus Spenden oder Steuergeldern sogenannter «entwickelter» Länder stammen und von privaten Stiftungen und Vereinen, kirchlichen Trägern, Regierungs- und Nichtregierungsorganisationen verwaltet werden. Diese Art globaler Mikrofinanzierung wurde bisher meistens als uneigennützige Wohlfahrt, Entwicklungshilfe oder partnerschaftliche Zusammenarbeit begriffen (vgl. Byström 2006: 1). Seit einigen Jahren bildet sich allerdings ein Gegendiskurs heraus, der im Mikrofinanzwesen einen neuen vielversprechenden Markt für private profitorientierte Investoren sieht (vgl. Matthäus-Maier 2008: IX). Die Deutsche Bank etwa prognostiziert mindestens eine Verzehnfachung des Investitionsvolumens in den nächsten 10 bis 20 Jahren, im Speziellen durch internationale private Investoren (Deutsche Bank Research 2008: 1; vgl. Callaghan et al. 2007: 118; vgl. Matthäus-Maier 2008: VIII). Und es zeichnen sich offenbar schon entsprechende praktische Veränderungen $\mathrm{ab}$. So konstatiert beispielsweise das Wall Street Journal unter Berufung auf Zahlen der Weltbank, dass im Jahr 2008 14,8 Milliarden US-Dollar Fremdkapital im Mikrofinanzwesen angelegt worden seien - 24\% mehr als im Vorjahr, und erstmals vorwiegend aus den Händen privater Investoren, nicht von Regierungen (Wall Street Journal 2010: o.S.).

Die in Diskurs und Praxis beobachtbare Transformation des Mikrofinanzwesens in einen globalen Markt lohnt in vielerlei Hinsicht eine soziologische und kulturanthropologische Untersuchung. Aus dem laufenden Forschungsprojekt heraus wird in diesem Essay beispielhaft ein bestimmtes Finanzierungsmodell be- 
sprochen, das verschiedenen ökonomischen Experten zufolge für private Investitionen in Mikrofinanzprojekte besonders geeignet

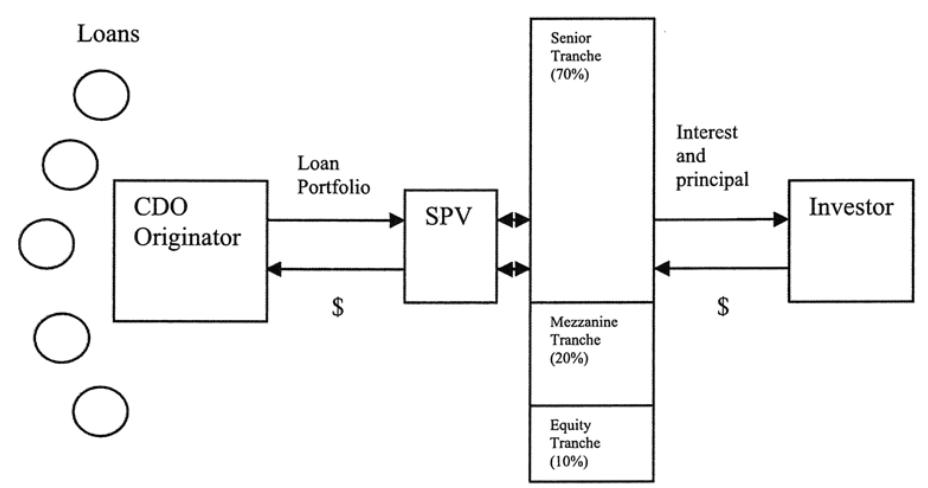

Eine typische CDO-Struktur nach Byström (2006: 22)

ist: die «collateralized debt obligation», abgekürzt «CDO» (Byström 2006; Financial Times 2009: o.S.; CGAP 2008: 7).

Auf der linken Seite der Abbildung sind die Kleinkredite dargestellt («Loans»). Die Kredite werden von einer Mikrofinanzinstitution vor Ort ausgegeben («CDO Originator»). Sie hält ein «Portfolio» aller dieser Einzelkredite, welches unter einer eigenen Rechtsform (SPV, d.h. "Special Purpose Vehicle») in verschiedene Risikoportionen zerlegt werden kann («Senior Tranche», «Mezzanine Tranche», «Equity Tranche»). Unter den Investoren, die auf der rechten Seite dargestellt sind, können dann die risikoscheuen ihr Geld eher in Wertpapiere der «Senior Tranche», die risikofreudigeren ihres in Papiere der «Equity Tranche» anlegen (vgl. Byström 2006: 5).

CDOs sind zuletzt in die Kritik geraten, da sie auf dem amerikanischen Hypothekenmarkt, von dem die jüngste Finanzkrise massgeblich ausging, sehr verbreitet waren (vgl. Sinn 2010: 169 ff.; McKenzie 2008; Heldt 2010: o.S.; Jobst 2008: 8). Verschiedene Finanzexperten meinen dagegen, dass mit CDOs im Fall von Mikrofinanzinvestitionen nicht nur finanzieller Gewinn zu erzielen sei, sondern sogar sozial Gutes geleistet werde; es wird hier von einer «doppelten» oder «dualen» Rendite gesprochen (Financial Times 2009: o.S.; Deutsche Bank 2008: 18; CGAP 2008: 2; CERISE 2010). Byström beispielsweise meint: 
By directly targeting the poor and therefore circumventing inefficient or perhaps even corrupt government structures, microlending could be an efficient means of doing well (financially) by doing good (socially). [...] Recently introduced financial instruments and vehicles [...] could make it possible for investors to tailor their risks and returns as they please. [...] [T] he MFIs [microfinance institutions] can simply serve as middle-hands and channel the risk and return of the pooled loan portfolio to a third party in the form of well defined standardized securities» (Byström 2006: 3, 4; Hervorhebungen BG).

Dieses Zitat wirft einige Fragen auf: Ist es in der Tat möglich, über Investitionen in der Art von CDOs Arme «direkt» zu erreichen? Lässt sich Armut ohne Weiteres bestimmen? Können lokale Bedingungen wie etwa Regierungsstrukturen in den Ländern, in denen Arme leben, einfach «umgangen» werden? Lassen sich die Risiken und Gewinne derart «masschneidern», dass sie den Investoren so passen, "wie es ihnen gefällt»? Können die Risiken und Gewinne von den Mikrofinanzinstitutionen, die die Kredite jeweils vor Ort vergeben, reibungs- und verlustfrei an Dritte übermittelt werden ("channel»), in der Gestalt "gut definierter» Wertpapiere? Jobst, ein früherer Mitarbeiter des IWF, argumentiert so ähnlich wie Byström: CDOs im Mikrofinanzbereich könnten sogar sehr illiquides Pfand in handelbare Wertpapiere «transformieren» und sonstige örtliche bis nationale Unsicherheiten «in Zaum halten» bzw. «umgehen» (Jobst 2008: 10, 11).

Die obige Abbildung suggeriert in der Tat einen solchen direkten, massgeschneiderten, reibungslosen und verlustfreien sowie gut definierten Investitionsprozess. Nachdem ein Portfolio in verschiedene Risikoportionen zerlegt wurde, können scheinbar einfach von rechts nach links die Anlagegelder fliessen («\$》), von links nach rechts die aggregierten Kreditinformationen («Loan Portfolio») und die Rückzahlungen («Interest and principal»). Ist dies nicht eine überschaubare globale Kreditbeziehung, bei dem die Armen (auf der linken Seite) sozial, die Investoren (auf der rechten Seite) finanziell profitieren?

Allerdings merkt Byström selbst an, dass einige «Hürden auf dem Weg» zu überwinden seien (2006: 3). Beispielsweise gebe es im Vergleich $\mathrm{zu}$ anderen Anlagebereichen eine «riesige Informationslücke» zwischen der Mikrofinanzinstitution und den Investoren (2006: 15). Während nämlich «gewöhnliche CDO-Geschäfte» zwischen «relativ ähnlichen Finanzinstitutionen mit ähnlicher Information, zum Beispiel Wall-Street-Investmentbanken», 
abgeschlossen würden, gebe es bei Mikrofinanz-CDOs ganz unterschiedliche Beteiligte - auf Seiten der Investoren etwa «reiche Individuen, kommerzielle Banken, Pensionsfonds und Regierungsorganisationen» (a.a.O.).Viele dieser Investoren könnten das Kreditrisiko wesentlich schlechter abschätzen als die jeweilige Mikrofinanzinstitution, die die Kredite vor Ort vergibt (a.a.O.).

\section{Zugang}

An dieser Problematik kann ein soziologisch-kulturanthropologisches Forschungsprogramm ansetzen. Der Investitionsprozess kann als komplexer Vertrauensprozess begriffen werden. Die erwähnte «Informationslücke» etwa ist der Annahme nach kein quasi technisch zu behebendes, einfaches Problem, sondern ein Mangel lebensweltlicher Vertrautheit. Es ist also ein umfangreiches kulturelles Phänomen. Dieses muss, so wird weiter angenommen, durch stellvertretendes Vertrauen in andere und anderes ausgeglichen werden. In Bezug auf die obige Abbildung kann folgende allgemeine These formuliert werden:

Die Abbildung ist nur eine "dünne», keine «dichte» Beschreibung (Geertz 1983: 11-12). Der globale Investitionsprozess ist in Wirklichkeit ein kompliziertes "Bedeutungsgewebe», in das sehr viele Menschen an verschiedenen Orten mit verschiedensten Weltsichten «verstrickt» sind (Geertz 1983: 9, 15). Dieses Gewebe ist u.a. aus diversen, kreuz und quer verlaufenden, zum Teil überlappenden Formen von Vertrauen geknüpft. Die Abbildung sollte durch eine Beschreibung der verschiedenen Bedeutungen (z.B. was für wen «sozial» heisst) und Weltsichten (von asiatischen, lateinamerikanischen oder afrikanischen Kreditnehmern, aber genauso von Bankexperten oder Investoren) sowie dieserVertrauensdichte ersetzt werden.

Nachfolgend werden beispielhaft drei vermuteteVertrauensformen im CDO-Investitionsprozess skizziert. Zunächst wird die Frage aufgeworfen, was genau sich in der Realität hinter den fünf mit «Loans» bezeichneten Kreisen verbirgt, die für die Kreditnehmer stehen (in der Abbildung links). 


\section{Vertrauen in die Vertrautheiten anderer}

Labie hat darauf hingewiesen, dass Armut und die entsprechenden Finanzierungsangebote, die eine einzelne Mikrofinanzinstitution auf der Ebene eines einzelnen Dorfes realistischerweise machen kann, schon für diese eine Institution schwer zu bemessen sind (vgl. Labie 2004: 12). «Armut» ist schon auf Mikro-Interaktionsebene eigentlich ein komplexes und dynamisches soziales Phänomen. Nach Gupta etwa müssten Arme als «soziale Akteure» verstanden werden, die ihre Situation kontextabhängig und im Zeitverlauf (historisch) ganz unterschiedlich deuten; auch werde Armut häufig als «statische Bestandsgrösse» aufgefasst, während es doch eine «dynamische Flussgrösse» sei (Gupta 2009: 134-136).Von Käufeler lässt sich lernen, dass es keine objektive Lösung für dieses Problem der Bemessung des Armen, Sozialen, Guten gibt - denn selbst die Ethnologen, deren Aufgabe es sei, «intim vertraut zu werden mit einer fremden Welt», hätten ständig mit deren Undurchsichtigkeit zu kämpfen (Käufeler 2008: 169). In manchen Gesellschaften würden gerade die finanziellen Verhältnisse als «Intimzonen» gelten; und selbst wenn dann Informationen «unter dem Siegel der Vertraulichkeit» mitgeteilt würden, so sei dies zwar «ein Indiz ihrer Bedeutung», jedoch «keine Garantie dafür, dass es wahr ist» (Käufeler 2008: 173, 177).

Die Bankexperten, die ein «Special Purpose Vehicle» verwalten (siehe Mitte der Abbildung), und die Investoren können sich vermutlich nicht wie Ethnologen auf mehrmonatigen Feldforschungen in die Ambivalenzen einer Lebenswelt einarbeiten. Es ist anzunehmen, dass sie stattdessen darauf vertrauen müssen, dass andere an ihrer Stelle eine solche Arbeit geleistet haben. Dafür, dass es solch ein stellvertretendes Vertrauen gibt, besteht ein erster empirischer Hinweis: Der Leiter eines noch relativ neuen Luxemburger Fonds für Mikrofinanzinvestitionen teilte im Interview (6.2.2010) mit, sein Fonds lasse sich von einer Nichtregierungsorganisation (NGO) beraten, die bereits neun Jahre mit Mikrofinanzinstitutionen zusammengearbeitet habe. Diese «Kernerfahrung» der NGO, wie er sich ausdrückte, nutze der Fonds, um passende Anlageprodukte zu entwickeln. In diesem Fall steht die NGO also vermutlich einen Schritt näher an der Lebenswelt der Kreditnehmer als der Fonds selbst.

Jobst berichtet allerdings, dass in vielen CDO-Strukturen nicht nur eine Mikrofinanzinstitution mit einer überschaubaren Zahl an Kreditbeziehungen eingebunden ist, wie die obige Abbildung suggeriert, sondern eine Reihe von Institutionen, die auch noch über die ganze Welt verteilt sind (vgl. Jobst 2008: 11). Es geht also empirisch 
nicht nur um eine Vertrautheit mit einer fremden Lebenswelt, sondern um diverse Vertrautheiten mit diversen fremden Lebenswelten. Vielleicht gibt es dann auch entsprechend vielfaches stellvertretendes Vertrauen? Auch stellt sich die Frage, ob und wenn ja, wie genau diese immense qualitative Vielfalt in eindeutige Wertpapiere mit klaren Risikoportionen übersetzt werden kann. Dies führt zur nächsten Vertrauensform:

\section{Systemvertrauen in Kalkulationen, erweitert um Kreativität}

Mennicken sagt, dass die Verfahren, die Banken zur Kalkulation des Kreditrisikos und also eigentlich zur Eindämmung von Unsicherheit einsetzen, neue Unsicherheiten schaffen: Zahlen sprächen nie für sich, es gebe immer Raum für verschiedene Interpretationen (Mennicken 2000:38). Sie fragt daraufhin, wie sich dann die Existenz solcher Verfahren erklären lasse, wenn nicht durch ihre (vermeintliche) inhärente Effizienz (a.a.O.). Der Grund sei eine langsam herangewachsene Selbstverständlichkeit («taken-for-grantedness»), also eine Institutionalisierung von Kalkulationen unter ökonomischen Akteuren (a.a.O.). In den Worten Luhmanns handele es sich um «Systemvertrauen», also eine (historische) Verlagerung von persönlichem Vertrauen in abstrakte Regelsysteme (Mennicken 2008: 39; Luhmann 1968/2000: 60 ff.).

In Anlehnung an Svetlova (2008) lässt sich gleichwohl vermuten, dass Bankexperten nicht bloss «blind» einer fixen Anzahl von Kalkulationsregeln vertrauen und folgen. Sie hat herausgearbeitet, dass Finanztheorien, die von Wirtschaftswissenschaftlern entwickelt wurden, von Portfoliomanagern in der Praxis nicht eins zu eins übernommen, sondern kreativ adaptiert werden (Svetlova 2008: 122). Es bleibt also zu untersuchen, wie sich das Systemvertrauen in Kalkulationen tatsächlich in der Praxis äussert, und wie es sich zur o.g. Problematik multipler Lebenswelten verhält: Wie genau, wenn überhaupt, überführen denn Bankexperten die Komplexität der Lebenswelten auf Kreditnehmerseite in verschiedene Risikoportionen, nämlich in prozentual jeweils verschieden grosse «Senior-», «Mezzanine-» und «Equity»-Tranchen? Werden kreative Spielräume genutzt, vielleicht auch unter den Kollegen debattiert? Inwiefern ist also die Konstruktion von Wertpapieren ein wesentlich komplexererVorgang mit einer viel grösseren Eigendynamik, als die 
einfachen Pfeile und Kästchen in der Mitte der obigen Abbildung suggerieren?

\section{Interaktiv hergestelltes Vertrauen}

Die irgendwie kalkulierten Wertpapiere müssen am Ende verkauft werden (siehe das Kästchen «Investor» auf der rechten Seite der Abbildung). Man stelle sich nun beispielsweise ein Verkaufsgespräch in einer Bank vor, in dem ein Bankangestellter gegenüber einem potentiellen Investor für eine Mikrofinanz-CDO wirbt. Mitten in der Erklärung der verschiedenen Risikotranchen setzt er sich plötzlich eine rote Clownsnase auf, steigt auf seinen Stuhl, holt eine Handvoll Bälle hervor und beginnt zu jonglieren. Dann singt er ein Lied, verbeugt sich und tritt $a b$.

Wird der Investor sich für den Kauf eines Wertpapiers entscheiden? Nach Garfinkel wäre das sehr unwahrscheinlich, denn der Bankmitarbeiter hat gegen eine Reihe von sozial erwartbaren, vertrauensbildenden Merkmalen einer Interaktion in einer Bank verstossen (Garfinkel 1967: 172-173). Diese Merkmale betreffen u.a. Kleidung, Mimik, Gestik, Körperhaltung und Rhetorik eines professionell anmutenden ökonomischen Experten sowie die technischen Hilfsmittel, derer sich dieser zu bedienen hat. In Bezug auf die Hilfsmittel beispielsweise wären anstelle von Jonglierbällen eher ein Notebook, Tabellen und Grafiken mit Zahlenkolonnen zu erwarten. Gibt es darüber hinaus im Fall von Mikrofinanz-CDOs, die anders als andere Anlageprodukte ja offenbar an sehr verschiedene Investoren verkauft werden (vgl. Byström 2006: 3), verschiedene vertrauensbildende Massnahmen in Verkaufsgesprächen? Werden zum Beispiel gegenüber Vertretern kommerzieller Banken, die ja eine vergleichbare Fachsprache sprechen, vor allem die Kalkulationen selbst diskutiert, während z.B. gegenüber Individuen ohne Bankausbildung in einfachen Narrativen und Metaphern ohne Zahlen gesprochen wird? Und wie genau wird, wenn überhaupt, jeweils über das «Soziale», über Armut, über das vermeintlich «Gute», also die komplexen kulturellen Phänomene am anderen Ende des Investitionsprozesses, gesprochen? Wird das grundsätzliche Problem der mangelnden Vertrautheit(en) mit der Lebenswelt bzw. den Lebenswelten auf der Seite der Kreditnehmer vielleicht in manchen Fällen «gelöst» durch rein situativ, rein interaktiv im Verkaufsgespräch selbst erzeugtes Vertrauen? 


\section{Schluss}

Das Mikrofinanzwesen sogenannter «Entwicklungsländer» scheint sich in einen globalen Markt zu transformieren. Als Beispiel wurden hierzu CDO-Investitionsstrukturen angeführt:Sie sind aus Sicht vieler Finanzexperten geeignet, um Kleinkredite als neuen Anlagebereich für private Investoren weltweit zu erschliessen. Zusätzlich zur finanziellen Rendite scheint in diesem Bereich eine «soziale» Rendite möglich zu sein: Über den Kauf von CDO-Wertpapieren am einen Ende der Welt kann einigen Autoren zufolge am anderen Ende der Welt ein Beitrag zur Armutsminderung geleistet werden. Diese Verknüpfung von Finanztermini mit den Begriffen des Sozialen, der Armut oder auch des Guten, so als gäbe es global keine divergierenden Lebenswelten, fordert eine kulturanthropologische und soziologische Untersuchung der betreffenden Finanzierungsstrukturen geradezu heraus. Wie eine solche Untersuchung aussehen kann, wurde hier kurz skizziert. Ein CDO-Investitionsprozess mutet auf den ersten Blick einfach und überschaubar an - eine am Prinzip der dichten Beschreibung orientierte Analyse wird aber die eigentliche Komplexität und Unsicherheit dieses Prozesses herausarbeiten können, so die hier vertretende Annahme. Es wurden drei Varianten von Vertrauen, die vermutlich an verschiedenen Stellen bzw. in unterschiedlicher Weise in die Konstruktion und den Verkauf von CDOWertpapieren hineinspielen, vorgestellt. $\mathrm{Ob}$ und wie genau diese drei Vertrauensformen den globalen Mikrofinanzmarkt in der Praxis mit-konstituieren, und ob es weitere relevante Vertrauensformen gibt - etwa globalisiertes Systemvertrauen in bestimmtes Geld, beispielsweise den US-Dollar (vgl. Luhmann 1968/2000: 63) - ist noch eingehend empirisch zu untersuchen.

\section{Literatur}

Byström H. (2006): The Microfinance Collateralized Debt Obligation: a Modern Robin Hood. http://www.nek.lu.se/publications/workpap/ Papers/WP06_14.pdf (Zugegriffen am 30. Mai 2010).

Callaghan I., Gonzalez H., Maurice D. (2007): Microfinance - On the Road to Capital Markets. Journal of Applied Corporate Finance 19: 115-123.

CERISE (2010): The Relationship Between Social and Financial Performance in Microfinance. http://www.sdc-employment-income. ch/en/Home/Financial_Sector/FSD_Main_Topics_and_Resources/ Social_Performance_Mainstreaming (Zugegriffen am 30. Mai 2010). 
CGAP (2008): Foreign Capital Investment in Microfinance. Balancing Social and Financial Returns. CGAP Focus Note.

Heldt C. (2010): Definition: Verbriefung von Kreditportfolios. Wirtschaftslexikon Gabler. http://wirtschaftslexikon.gabler.de/Definition/verbriefung-von-kreditportfolios.html (Zugegriffen am 30. Mai 2010).

Deutsche Bank Research (2008): Mikrofinanz-Investments: Eine sozial verantwortliche Anlage mit großem Potenzial. Frankfurt am Main: Deutsche Bank Research.

Financial Times (2009): Microfinance Group in CDO Scheme. http:// www.finca.org/atf/cf/\%7BF69F69E6-275A-4FA1-BC75649E1EDCD1A4\%7D/Financial\%20Times\%20Article.pdf (Zugegriffen am 30. Mai 2010).

Garfinkel H. (1967): Studies in Ethnomethodology. Englewood Cliffs: Prentice-Hall.

Geertz C. (1983): Dichte Beschreibung. Bemerkungen zu einer deutenden Theorie von Kultur. In: Dichte Beschreibung. Beiträge zum Verstehen kultureller Systeme. Frankfurt am Main: Suhrkamp.

Gupta A. (2009): Nationale Armut, globale Armut und Neoliberalismus: Eine anthropologische Kritik. In: Büschel H., Speich D. (Hg.): Entwicklungswelten. Globalgeschichte der Entwicklungszusammenarbeit, Frankfurt: Campus: 113-139.

Jobst AA. (2008): Unscathed Securitisation: Emerging Market CDOs After the US Sub-prime Mortgage Crisis. In: Deutsche Bank (Hg.): Global Securitisation and Structured Finance, http://www.globalsecuritisation. com/ (Zugegriffen am 30. Mai 2010).

Käufeler H. (2008): Vertrauen, Vertrautheit, Vertraulichkeit. Über den Umgang mit Intimität in der Ethnologie. In: Tomkowiak I., Egli W. (Hg.): Intimität, Zürich: Chronos: 169-183.

Labie M. (2004): Microfinance: un Etat des Lieux. Monde en Développement 2: 9-23.

Luhmann N. (1968): Vertrauen. Ein Mechanismus der Reduktion sozialer Komplexität. Stuttgart: Lucius und Lucius.

Matthäus-Maier I. (2008): Preface: New Partnerships for Innovation in Microfinance. In: New Partnerships for Innovation in Microfinance. Berlin: Springer:VII-XI.

MacKenzie D. (2008): End-of-the-World Trade. London Review of Books 30: 24-26.

Mennicken A. (2000): Figuring Trust: The Social Organization of Credit Relations. In: Kalthoff H., Rottenburg R., Wagener HJ. (Hg.): Facts 
and Figures: Economic Representations and Practices, Ökonomie und Gesellschaft, Marburg: Metropolis: 35-58.

Sinn HW. (2009): Kasino-Kapitalismus: wie es zur Finanzkrise kam, und was jetzt zu tun ist. Berlin: Econ.

Svetlova E. (2009): Theoretical Models as Creative Resources in Financial Markets. In: Jansen SA., Schröter E., Stehr N. (Hg.): Rationalität oder Kreativität? Multidisziplinäre Beiträge zur Analyse der Produktion, Organisation und Bildung von Kreativität, Wiesbaden:VS: 121-135.

Wall Street Journal (2010): Microfinance‘s Midlife Crisis. http://online. wsj.com/article/SB10001424052748703315004575073510472268430. html?mod=googlenews_wsj (Zugegriffen am 30. Mai 2010).

- Dr. des Barbara Grimpe arbeitet im Rahmen des Projekts «Vertrauen verstehen» an einer Habilitation mit dem Thema «Die Expansion von Mikrofinanzen aus den ökonomischen Nischen der Entwicklungszusammenarbeit der 1970er-Jahre in die heutige Wall Street Finance». 